\title{
Gait transition dynamics are modulated by concurrent cognitive activity
}

\author{
Mohammad Abdolvahab ${ }^{1}$
}

Published online: 20 June 2015

(C) The Psychonomic Society, Inc. 2015

\begin{abstract}
In tasks with two categorically distinct behavioral possibilities a person beginning with one option will typically switch to the other at a higher value of a control parameter in an ascending (increasing) sequence than in a descending (decreasing) sequence. For example, the switch from walking to running on an accelerating treadmill occurs at a higher speed than the switch from running to walking on a decelerating treadmill. The reported research posed the question of whether this variant of behavioral hysteresis was affected by concurrent cognitive activity. Participants walked or ran on a treadmill with a constant acceleration or deceleration while counting backwards by sevens or ones, or not counting. The degree of hysteresis, the difference between walk-to-run and run-to-walk transition speeds, increased with cognitive difficulty. Specifically, the increased hysteresis was shown to be due to lower run-to-walk transition speeds for the more difficult concurrent cognitive tasks. These results support the hypothesis that cognitive load occupies attentional resources that contribute to triggering human gait transitions.
\end{abstract}

Keywords Gait transition · Hysteresis · Dynamical systems · Dual-task methodology

Mohammad Abdolvahab

mohammad.abdolvahab@uconn.edu

1 Center for the Ecological Study of Perception and Action, Department of Psychology, University of Connecticut, 406 Babbidge Rd, Storrs, CT 06269, USA

\section{Introduction}

Many everyday actions occur in the context of other activities, including those that are relevant to accomplishing a task and those that are irrelevant to that task, actions that might demand attention and cognitive resources and constrain perception or motor performance. In experimental psychology, dual-task methodology, ${ }^{1}$ which requires participants to perform two or more concurrent activities, has been extensively used in various paradigms. A subset of these studies examines the attentional demands of motor tasks. For example, Kerr, Condon, and McDonald (1985) found that a concurrent standing balance task disrupted recall on a spatial memory task. The authors attributed this finding to a reliance of postural control and cognitive spatial processing on common central neural mechanisms. Lajoie, Teasdale, Bard, and Fleury (1993) demonstrated longer reaction times to auditory stimuli when participants stand and walk than when they sit. Also, Pellecchia (2005) found that postural sway increased when a cognitive task was performed concurrently with a postural task.

In the study of gait control, in particular, there are also studies related to the dual-task application. Most existing dual-task applications to the study of gait, however, have been to demonstrate the increased attentional resources needed for gait control (in the elderly, e.g., Brown, Shumway-Cook, \& Woollacott, 1999; Lajoie, Teasdale, Bard, \& Fleury, 1996; Marsh \& Geel, 2000, or in patient populations such as those with Parkinson's and Alzheimer's disease, e.g., Morris, Iansek, Smithson, \& Huxham, 2000; Morris, Iansek, Summers, \&

\footnotetext{
${ }^{1}$ To examine attentional demands and motor performance in adults, investigators have used various cognitive activities to manipulate task difficulty, including numeric tasks, Brooks spatial and non-spatial memory tasks (Maylor \& Wing, 1996; Maylor, Allison, \& Wing, 2001; Stelmach, Zelaznik, \& Lowe, 1990), or varied rates of presenting word lists for memorizing (e.g., Li, Lindenberger, Freund, \& Baltes, 2001).
} 
Matyas, 1995; Camicioli, Howieson, Lehman, \& Kaye, 1997; see also Abernethy, Hanna, \& Plooy, 2002). Ebersbach, Dimitrijevic, and Poewe (1995) suggested that although walking is a highly practiced task, gait parameters related to control of balance are subject to modifications according to the attentional demand of concurrent tasks. Daniels and Newell (2003) examined walk-to-run transitions when participants walked on an accelerating treadmill. They found that walk-to-run transition speeds increased when the participants solved mental arithmetic at two levels of difficulty.

The present study was aimed at studying the effect of concurrent cognitive activity on the dynamics of human adults' walk-to-run (WR) and run-to-walk (RW) transitions on a treadmill and in particular the phenomenon known as hysteresis. Hysteresis is one of the characteristics of nonlinear phase transitions. Hysteresis has a long history in inanimate systems and has, in recent years, been variously reported in different animate systems. In particular it has been reported in a number of human perception-action experiments (e.g., Fitzpatrick, Carello, Schmidt, \& Corey, 1994; LoprestiGoodman, Richardson, Baron, Carello, \& Marsh, 2009; Richardson, Marsh, \& Baron, 2007; Tuller, Case, Ding, \& Kelso, 1994; Van der Kamp, Savelsbergh, \& Davis, 1998). Diedrich and Warren (1995) provided a dynamical systems account for human gait transition (with walking and running as two attractive states of the system) and demonstrated that gait transitions satisfy the criteria of nonlinear phase transitions - specifically, they exhibit hysteresis, that is, higher transition speed for WR in a sequence of increasing treadmill speeds than for RW in a sequence of decreasing treadmill speeds. $^{2}$

It has been suggested that concurrent cognitive activity affects hysteresis. For example, Lopresti-Goodman et al. (2009) discovered exaggerated hysteresis with increased cognitive task difficulty in perception of the affordance of graspability. For instance, when participants performed the concurrent cognitive task of counting backward from a three-digit number by sevens more hysteresis was observed than when they performed the simultaneous arithmetic task of counting backward by ones. Figure 1 shows a hypothetical schema of such an exaggeration of the hysteresis in the context of human gait transitions.

There are enough hints (summarized above) to suggest that imposing cognitive constraints upon walking or running suffices to change their dynamics. For example, Getchell and Whitall (2004) showed that intentional switching between gaits from different symmetry classes (e.g., walk vs. gallop)

\footnotetext{
${ }^{2}$ For other similar demonstrations of hysteresis in human locomotion see Abdolvahab (2014, 2015); Hreljac (1995); Hreljac, Imamura, Escamilla, and Edwards (2007); Li (2000); and Turvey, Holt, LaFiandra, and Fonseca (1999).
}

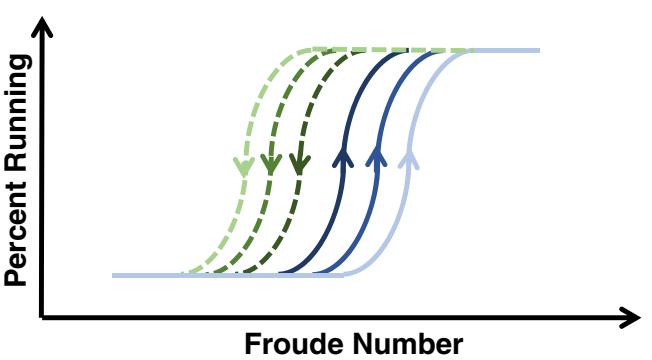

Fig. 1 Schematic representation of exaggeration in hysteresis in gait transition by concurrent cognitive activity. The darkest loop corresponds to no concurrent cognitive activity; the solid line represents an ascending sequence or increasing the speed, while the dashed line represents a descending sequence or decreasing the speed. With the addition of concurrent cognitive activity of increasing difficulty, the area inside the hysteresis loop increases. Lighter lines represent the "easier" mental arithmetic task (counting backward by ones), the lightest lines correspond to the "harder" mental arithmetic task (counting backward by sevens). The larger area between the lighter trajectories corresponds to the more exaggerated hysteresis

resulted in changes in dynamical hysteresis. ${ }^{3}$ In the present experiment, extending the findings of Daniels and Newell (2003), the effect of conducting a simple cognitive activity in parallel with walking and running has been studied. Specifically, it has been hypothesized that concurrent cognitive activity may affect the WR or RW transition speeds and consequently the degree of hysteresis in ordinary bipedal locomotion.

\section{Method}

\section{Participants}

Six women and nine men (mean age $=23.1 \pm 3$ years; mean leg length $=89 \mathrm{~cm}$ ) from the University of Connecticut participated in this experiment. Participants were paid at a rate of US $\$ 10$ per hour for their participation. The university's Institutional Review Board approved all procedures.

\section{Materials}

A Trackmaster TMX-425 treadmill (180-kg capacity, running surface of $76 \times 160 \mathrm{~cm}$, speed range $0.22-5.36 \mathrm{~m} / \mathrm{s}$ ) with a manual controller and safety siderails was used.

\footnotetext{
${ }^{3}$ With regard to the observation of negative hysteresis in the study by Getchell and Whitall (2004), it can be argued that in their experiment the constraint on the system was intentional rather than cognitive; the intentional switching between symmetric and asymmetric gaits (e.g., walk-togallop or run-to-gallop) can be taken as acting as a constraint on the system that can alter symmetries at the level of perception-action cycle (see Abdolvahab, Carello, Pinto, Turvey, \& Frank, 2015).
} 


\section{Procedure}

Each participant completed two repetitions of walk-to-run with increasing treadmill speed and two repetitions of runto-walk with decreasing treadmill speed for each of three cognitive conditions (no concurrent cognitive activity, counting backwards by ones and counting backwards by sevens). Prior to the experiment, each participant completed a practice trial for each of the two sequence conditions (ascending or descending). The participants were asked to avoid holding safety siderails unless necessary.

In the case of counting backwards, the participants did the task verbally and loudly. The performance sequence (walk-torun preceding run-to-walk or vice versa) and the different task conditions were counterbalanced across participants. The participant's leg length was defined by adding the measures of hip-to-knee and knee-to-ankle. The participants were tested on the treadmill with constantly increasing or decreasing speed at constant increments of $0.045 \mathrm{~m} / \mathrm{s}(0.1 \mathrm{mph})$ every $2 \mathrm{~s}$ (yielding a constant acceleration or deceleration of approximately $0.02 \mathrm{~m} / \mathrm{s}^{2}$ ). The final results were based on averaging across repetitions.

\section{Design and analysis}

The experiment employed a 2 (sequence: walk-to-run, run-towalk) $\times 3$ (condition: no counting, counting backwards by ones, counting backwards by sevens) within-subjects design. There were two repetitions for each trial. Hence, participants performed 12 trials in sum. The transition speeds were averaged across repetitions. The critical Froude numbers ${ }^{4}(\mathrm{Fr})$ were computed for each participant by dividing transition speed squared $\left(v^{2}\right)$ by the participant's leg length and gravitational acceleration $\left(F r=v^{2} / l g ; g=9.81 \mathrm{~m} / \mathrm{s}^{2}\right)$.

\section{Results}

The experimental results showed that participants switched from walking to running at larger Froude numbers in the ascending sequences $(0.46 \pm 0.08)$ than in the descending sequences $(0.33 \pm 0.06)$. A 3 (Task) $\times 2$ (Sequence) withinsubjects ANOVA on the mean Froude numbers revealed significant effects for Cognitive Task, $F(2,28)=7.2, p<0.01$, Sequence (Ascending vs. Descending), $F(1,14)=81.8$, $p<0.001$, and Sequence $\times$ Cognitive Task, $F(2,28)=13.8$, $p<0.001$. Hysteresis was least in the absence of a concurrent cognitive task $\left(\Delta F r_{\text {mean }}=0.07\right)$, intermediate when counting backward by ones $\left(\Delta F r_{\text {mean }}=0.13\right)$, and most when counting

\footnotetext{
${ }^{4}$ As applied here, the Froude number is a normalized dimensionless measure of speed for differently sized participants and represents the ratio between inertial and gravitational forces in human locomotion.
}
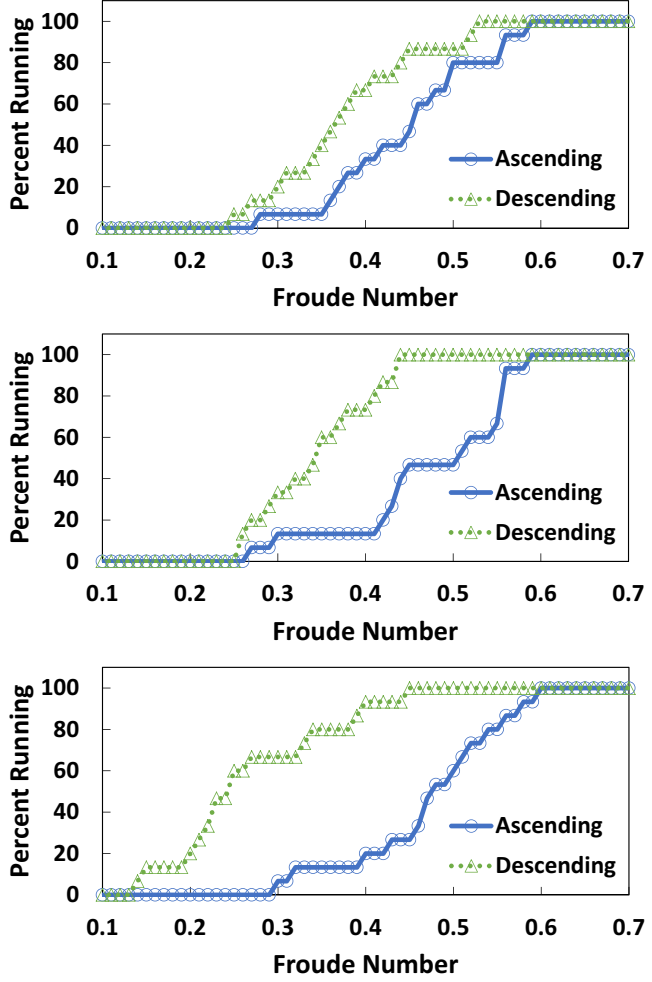

Fig. 2 Exaggeration in hysteresis with increase in the difficulty of concurrent cognitive task. Top panel: no concurrent cognitive activity. Middle panel: counting backward by ones. Bottom panel: counting backward by sevens

backward by sevens $\left(\Delta F r_{\text {mean }}=0.20\right)$. Figure 2 shows the percentage transition versus Froude numbers for the three conditions and the two sequences for each, ascending and descending. It is evident in Fig. 2 that the area between the two curves (for Ascending and Descending sequences) increases with the increasing cognitive task difficulty indicating an exaggerated hysteresis across the conditions.

Figure 3 depicts the mean critical $F r$ numbers for different conditions. As shown, mean critical Fr for ascending sequences increased from 0.45 for no concurrent cognitive task condition to 0.47 for both "easy" and "hard" task conditions.

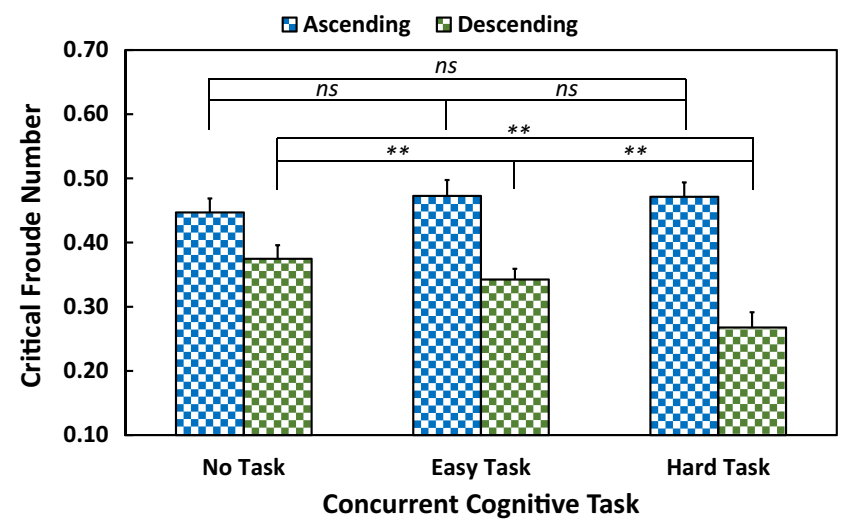

Fig. 3 Mean values of critical Froude numbers for task conditions (error bars represent standard error of the means) 
However, post-hoc paired mean comparisons of ascending sequences across task conditions did not reveal significant results. For descending sequences, mean critical $F r$ decreased from 0.37 for no concurrent task condition to 0.34 and 0.27 for "easy" and "hard" task conditions, respectively. Post-hoc paired mean comparisons of descending sequences showed significant results $(p<0.01)$ across all task conditions.

\section{Discussion}

The results of the experiment confirmed expectations about the dynamics of gait transition when participants are engaged in a concurrent cognitive task. The predictions followed from prior empiric observations of hysteresis in behavioral transitions (e.g., Lopresti-Goodman et al., 2009). The question of the present study was how concurrent cognitive activity might function as a constraint on bipedal dynamics, specifically, the hysteresis in transiting from walk to run and vice versa. It was predicted that increases in task difficulty would result in increases in the degree of hysteresis exhibited $(\Delta F r)$.

The results of the experiment were compatible, in part, with those reported in Daniels and Newell (2003). In their study, they examined the hypothesis that cognitive perceptual processes are involved in determining the WR transition in human locomotion. They recorded WR transition speeds and peripheral rating of perceived exertion of healthy participant walking on a treadmill with increasing speed while solving mental arithmetic at two levels of difficulty (easy and hard). Daniels and Newell observed that the transition speeds increased during both levels of mental arithmetic. They concluded that cognitive load distracts attentional focus from physiologic cues that contribute to triggering human gait transitions. In the present study, although only a mild increase in the WR transition values was observed, the cognitive load showed significant impact on the RW transition values. The overall results were also reported in terms of hysteresis. It was observed that hysteresis was significantly affected by concurrent cognitive activity.

Cognitive constraint can alter the intrinsic dynamics of the perceiver with regard to the environment. In dynamical systems theory, this alteration is in the form of relocation of attractors. The results of the present experiment are also comparable with the predictions with regard to impact of concurrent cognitive activity on the attractor dynamics (Pellecchia \& Turvey, 2001; Pellecchia, Shockley, \& Turvey, 2005). For instance, Pellecchia and Turvey (2001) inquired how the attractors of bimanual rhythmic coordination, given as the solutions of a motion equation in relative phase, were affected by cognitive activity. In their experiments, participants were required to swing two pendulums with their two hands in an in-phase or anti-phase manner while performing an information reduction task (Posner, 1964). They observed that cognitive activity amplified deviation from both in-phase $\left(0^{\circ}\right)$ and anti-phase $\left(180^{\circ}\right)$ to the same degree with amplification (in degrees), a linear increasing function of magnitude of information reduction (in bits). In the present study, even though the detuning was manifest in the critical $F r$ for RW transitions, the post-hoc analysis did not reveal symmetric changes for WR transitions. One can explain this observed phenomenon through the interplay between the attentional resources and biomechanical constraints. In WR transitions, in the presence of a concurrent counting task, participants tend to delay the transition from walk to run until the biomechanical limits are reached and transition to run is inevitable. In contrast, in RW transitions in the absence of biomechanic constraints, cognitive resources taken by the counting task, lead participants to delay transitioning from run to walk until the speed is so low that continuing running is more demanding than dealing with the transition and the associated attentional resources. Additionally, since the transitions are performed on a treadmill and the post-transition gait needs to be stabilized to reduce the risk of losing balance, participants delay the transition until the speed is low enough to comfortably stabilize post-transition gait. This interpretation is compatible with the prediction that the transition speeds are amplified by the difficulty of concurrent cognitive task.

Acknowledgments This work was conducted as part of the doctoral dissertation of the author while at the Center for the Ecological Study of Perception and Action at the University of Connecticut. The author is thankful to Prof. Michael Turvey for his guidance in conducting the study and help in preparing the present manuscript and to the anonymous reviewers for their helpful comments. The study was partially supported by NSF Grant BCS-1344725 (INSPIRE Track 1).

\section{References}

Abdolvahab, M. (2014). Positive hysteresis and negative hysteresis in human gait transition. Storrs: Doctoral Dissertation, University of Connecticut.

Abdolvahab, M. (2015). A synergetic model for human gait transitions. Physica A: Statistical mechanics and its Applications, 433, 74-83.

Abdolvahab, M., Carello, C., Pinto, C., Turvey, M. T., \& Frank, T. D. (2015). Symmetry and order parameter dynamics of the human odometer. Biological Cybernetics, 109(1), 63-73.

Abernethy, B., Hanna, A., \& Plooy, A. (2002). The attentional demands of preferred and non-preferred gait patterns. Gait \& Posture, 15(3), 256-265.

Brown, L. A., Shumway-Cook, A., \& Woollacott, M. H. (1999). Attentional demands and postural recovery: The effects of aging. The Journals of Gerontology Series A: Biological Sciences and Medical Sciences, 54(4), 165-171.

Camicioli, R., Howieson, D., Lehman, S., \& Kaye, J. (1997). Talking while walking The effect of a dual task in aging and Alzheimer's disease. Neurology, 48(4), 955-958.

Daniels, G. L., \& Newell, K. M. (2003). Attentional focus influences the walk-run transition in human locomotion. Biological Psychology, 63(2), 163-178. 
Diedrich, F. J., \& Warren, W. H., Jr. (1995). Why change gaits? Dynamics of the walk-run transition. Journal of Experimental Psychology: Human Perception and Performance, 21(1), 183-202.

Ebersbach, G., Dimitrijevic, M. R., \& Poewe, W. (1995). Influence of concurrent tasks on gait: A dual-task approach. Perceptual and Motor Skills, 81(1), 107-113.

Fitzpatrick, P., Carello, C., Schmidt, R. C., \& Corey, D. (1994). Haptic and visual perception of an affordance for upright posture. Ecological Psychology, 6(4), 265-287.

Getchell, N., \& Whitall, J. (2004). Transitions to and from asymmetrical gait patterns. Journal of Motor Behavior, 36(1), 13-27.

Hreljac, A. (1995). Effects of physical characteristics on the gait transition speed during human locomotion. Human Movement Science, 14(2), 205-216.

Hreljac, A., Imamura, R., Escamilla, R. F., \& Edwards, W. B. (2007). Effects of changing protocol, grade, and direction on the preferred gait transition speed during human locomotion. Gait \& Posture, 25(3), 419-424.

Kerr, B., Condon, S. M., \& McDonald, L. A. (1985). Cognitive spatial processing and the regulation of posture. Journal of Experimental Psychology: Human Perception and Performance, 11(5), 617-622.

Lajoie, Y., Teasdale, N., Bard, C., \& Fleury, M. (1993). Attentional demands for static and dynamic equilibrium. Experimental Brain Research, 97, 139-144.

Lajoie, Y., Teasdale, N., Bard, C., \& Fleury, M. (1996). Upright standing and gait: Are there changes in attentional requirements related to normal aging? Experimental Aging Research, 22(2), 185-198.

Li, L. (2000). Stability landscapes of walking and running near gait transition speed. Journal of Applied Biomechanics, 16(4), 428-435.

Li, K. Z., Lindenberger, U., Freund, A. M., \& Baltes, P. B. (2001). Walking while memorizing: Age-related differences in compensatory behavior. Psychological Science, 12(3), 230-237.

Lopresti-Goodman, S. M., Richardson, M. J., Baron, R. M., Carello, C., \& Marsh, K. L. (2009). Task constraints on affordance boundaries. Motor Control, 13(1), 69-83.

Marsh, A. P., \& Geel, S. E. (2000). The effect of age on the attentional demands of postural control. Gait \& Posture, 12(2), 105-113.

Maylor, E. A., Allison, S., \& Wing, A. M. (2001). Effects of spatial and nonspatial cognitive activity on postural stability. British Journal of Psychology, 92(2), 319-338.
Maylor, E. A., \& Wing, A. M. (1996). Age differences in postural stability are increased by additional cognitive demands. The Journals of Gerontology Series B: Psychological Sciences and Social Sciences, 51(3), 143-154.

Morris, M., Iansek, R., Smithson, F., \& Huxham, F. (2000). Postural instability in Parkinson's disease: A comparison with and without a concurrent task. Gait \& Posture, 12(3), 205-216.

Morris, M. E., Iansek, R., Summers, J. J., \& Matyas, T. A. (1995). Motor control considerations for the rehabilitation of gait in Parkinson's disease. Advances in Psychology, 111, 61-93.

Pellecchia, G. L. (2005). Dual-task training reduces impact of cognitive task on postural sway. Journal of Motor Behavior, 37(3), 239-246.

Pellecchia, G. L., Shockley, K., \& Turvey, M. T. (2005). Concurrent cognitive task modulates coordination dynamics. Cognitive Science, 29(4), 531-557.

Pellecchia, G. L., \& Turvey, M. T. (2001). Cognitive activity shifts the attractors of bimanual rhythmic coordination. Journal of Motor Behavior, 33(1), 9-15.

Posner, M. I. (1964). Information reduction in the analysis of sequential tasks. Psychological Review, 71(6), 491-504.

Richardson, M. J., Marsh, K. L., \& Baron, R. M. (2007). Judging and actualizing intrapersonal and interpersonal affordances. Journal of Experimental Psychology: Human Perception and Performance, 33(4), 845-859.

Stelmach, G. E., Zelaznik, H. N., \& Lowe, D. (1990). The influence of aging and attentional demands on recovery from postural instability. Aging Clinical and Experimental Research, 2(2), $155-161$.

Tuller, B., Case, P., Ding, M., \& Kelso, J. A. (1994). The nonlinear dynamics of speech categorization. Journal of Experimental Psychology: Human Perception and Performance, 20(1), 316.

Turvey, M. T., Holt, K. G., LaFiandra, M. E., \& Fonseca, S. T. (1999). Can the transitions to and from running and the metabolic cost of running be determined from the kinetic energy of running? Journal of Motor Behavior, 31(3), 265-278.

Van der Kamp, J., Savelsbergh, G. J., \& Davis, W. E. (1998). Body-scaled ratio as a control parameter for prehension in 5-to 9-year-old children. Developmental Psychobiology, 33(4), 351-361. 